\title{
Imaging Contrast with Multiple Ion Beams
}

\author{
Huimeng Wu, Sybren Sijbrandij, Shawn McVey and John Notte
}

Ion Microscopy Innovation Center, Carl Zeiss Microscopy LLC, One Corporation Way, Peabody MA 01960, USA

A commercial Ga-FIB/SEM system can directly image samples using an ion beam or e-beam before, after or during milling/depositing process. This capability provides important feedback for process control. Because of the limited spatial resolution and $\mathrm{Ga}$ contamination of the $\mathrm{Ga}^{+}$ion beam, the e-beam is often considered as the primary imaging tool. But ion beam imaging also provides important information about the samples. Orion Nanofab integrates $\mathrm{He}^{+}, \mathrm{Ne}^{+}$and $\mathrm{Ga}^{+}$focused ion beams on one single platform. $\mathrm{He}^{+}$and $\mathrm{Ne}^{+}$ion beams are based on the gas field ion source (GFIS) technology. The images generated by $\mathrm{He}^{+} / \mathrm{Ne}^{+}$ion beams are sub-nm in resolution capturing intricate details of the samples[1]. Nanofab also provides an optional state of art Ga-FIB. With this unique configuration, Orion Nanofab provides a great platform to study ion beam imaging with a variety of ion species.

In this study, we investigate three imaging modes: secondary electron (SE), secondary ion (SI) and backscattered ion (BSI), using three ion beams: $\mathrm{He}^{+}, \mathrm{Ne}^{+}$and $\mathrm{Ga}^{+}$. The most common imaging mode of the e-beam and ion beam is via SE. Compared with the SE image of e-beam, ion beam imaging is more sensitive to surface topography, easier charge neutralization with a flood gun, better for passive voltage contrast, and stronger grain orientation contrast due to ion-channeling effects. Other imaging modes via SI and BSI can provide additional information to better characterize the sample and eliminate the need for further analysis. SI images provide material contrast because of the different secondary ion yield of different materials, particularly sensitive to the presence of oxides and carbides. This property enables the studies of corrosion or grain boundary segregation in metallic systems[2]. Also the SI mode allows the electron flood gun to operate in continuous emission mode without multiplex imaging. BSI images provide less surface specification, but the atomic number "Z" contrast. Because BSI has the similar energy as the incident ion beam energy, the signal can pass through several layers.

The schematic illustration in Fig. 1a shows three ion beams on a Orion Nanofab and the ion beam/sample interactions. When an ion beam impinges on a sample surface, it emits secondary particles, including low energy secondary electrons, secondary ions, backscattered ions and others. An experimental measurement was carried out to compare the BSI signal and SI signal for $\mathrm{He}^{+}$and $\mathrm{Ne}^{+}$ion beams on ten chosen target elements (Fig. 1b). The results show that the $\mathrm{Ne}^{+}$induced SI signal is more than one order of magnitude larger than $\mathrm{He}^{+}$, as is expected from SRIM-calculations of sputter yield[3]. For a $\mathrm{He}^{+}$ion beam, the BSI signal is about an order of magnitude larger than the SI signal. For a $\mathrm{Ne}^{+}$ ion beam, the BSI signal intensity is approximately equal to the SI signal. From this measurement, a $\mathrm{He}^{+}$ ion beam is suitable for SE and BSI images, but the SI signal is relatively low. A $\mathrm{Ne}^{+}$ion beam is useful for acquiring all imaging with SE, SI and BSI signal.

Two samples were tested to show that the combination of SE, BSI and SI images can provide complementary information about the samples. Fig. $2 \mathrm{a}$ and $2 \mathrm{~b}$ are tilted $\mathrm{He}^{+}$ion beam images of carbon thin film on coper grid. The SE image (Fig. 2a) shows the surface topography of the thin carbon film and the SE signal from the materials beneath the carbon film can't penetrate through the film. The BSI image (Fig. 2b) shows better contrast of the underlying material than the carbon film because of the 
carbon low Z. Fig. 2c and 2d are $\mathrm{Ne}^{+}$ion beam images of the oxide patterns with Al pads. The SE image (Fig. 2c) shows the very strong voltage contrast. Two grounded Al pads on the bottom corner of the image are very bright. The two floating pads in the center of the image appear almost black. All oxide dielectric patterns are very dark because of charging. While in the SI image (Fig. 2d), all oxide dielectric patterns show good contrast and all four metal Al pads are dark because of the low SI yield. No flood gun was applied during the imaging of either samples.

\section{References:}

[1] BW Ward, JA Notte and NP Economou, Vac. Sci. B. 24 (2006), p. 2871.

[2] A Laquerre and MW Phaneuf, Microscopy \& Microanalysis, 14 (2008), p. 620.

[3] http://www.srim.org/

a

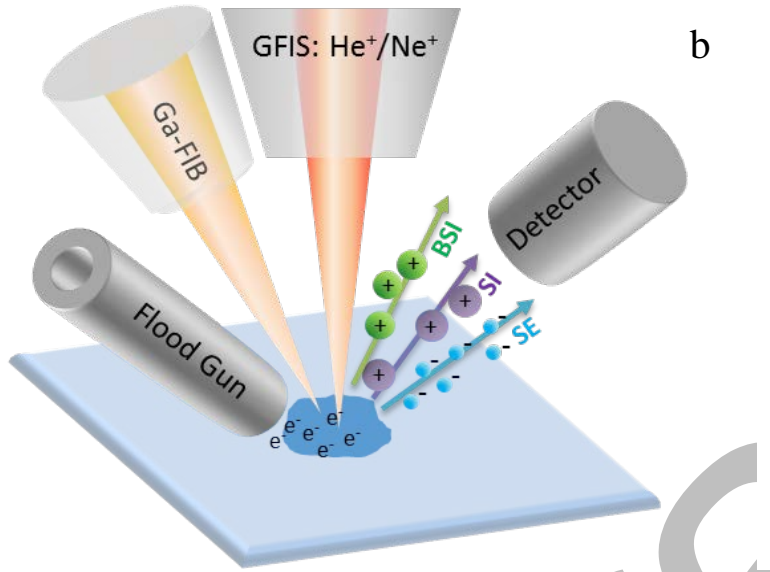

b

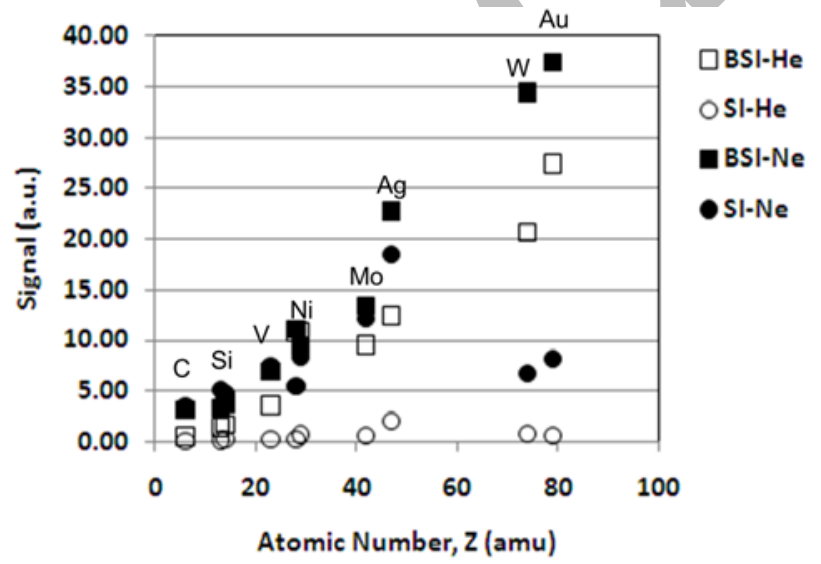

Figure 1. a) Schematic illustration of three ion beams on a) Orion Nanofab and the ion beam/sample interaction. b) Comparison of BSI and SI signal for $25 \mathrm{keV} \mathrm{He}^{+}$and $\mathrm{Ne}^{+}$ion beam.

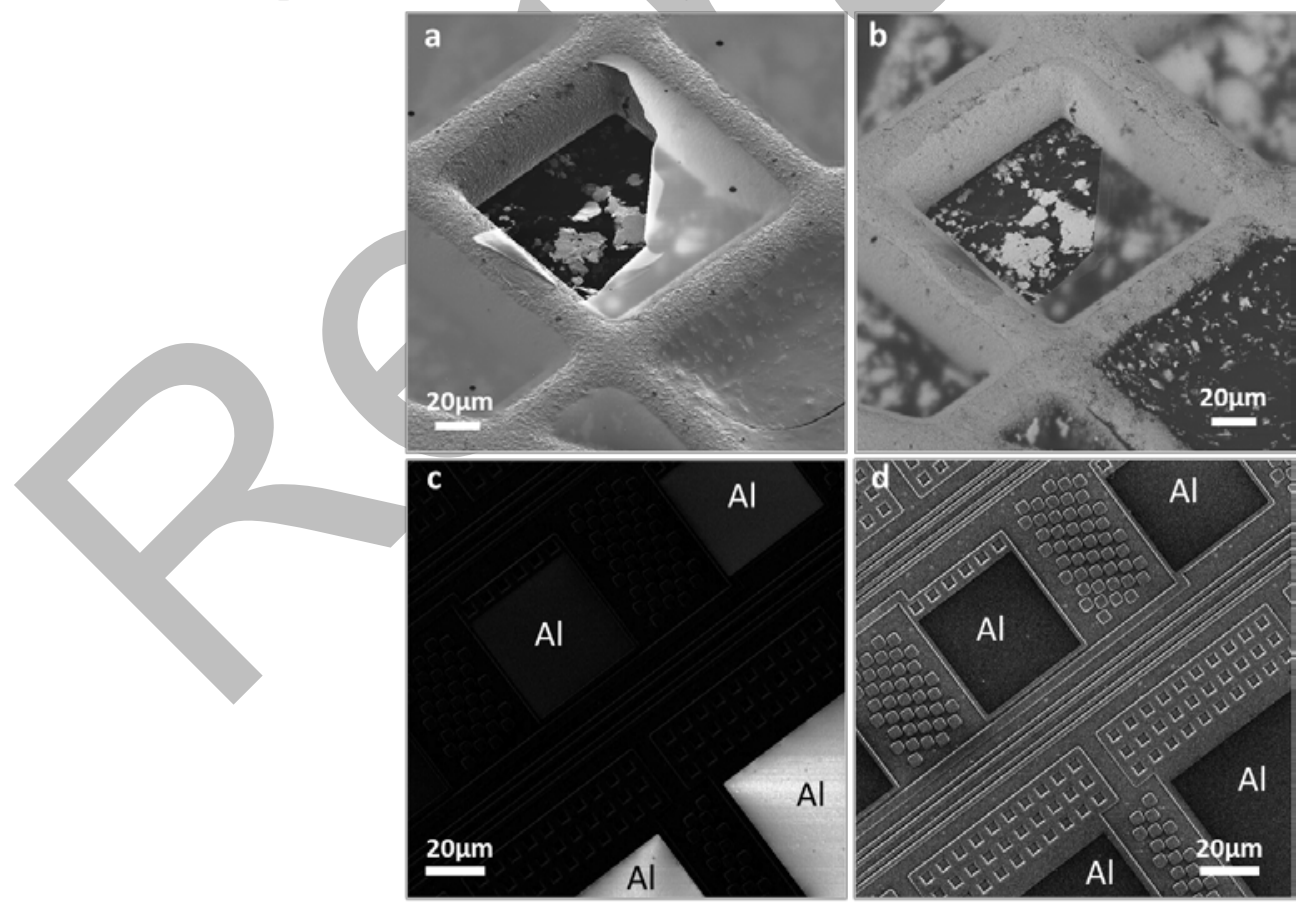

Figure 2. Tilted $\mathrm{He}^{+}$ion beam a) SE and b) BSI images of carbon thin film on coper grid. $\mathrm{Ne}^{+}$ion beam c) SE and d) SI images of the oxide patterns with Al pads. 